\title{
Reserva funcional adrenal en el espectro completo de la enfermedad renal crónica
}

\author{
René Rodríguez-Gutiérrez ${ }^{1,2}$, J. Gerardo González-González'1,2, Alberto Martínez-Rodríguez³, \\ Erick Burciaga-Jiménez², Ricardo César Solís², Alejandro Díaz González-Colmenero², \\ Luz Adriana Ramírez-García², Sofía Mariño-Velasco ${ }^{2}$ y Francisco J. Barrera-Flores² \\ ${ }^{1}$ Servicio de Endocrinología; ${ }^{2}$ Plataforma INVEST Medicina UANL-KER Unit Mayo Clinic (KER Unit México); ${ }^{3}$ Departamento de Medicina Interna. \\ Facultad de Medicina Y Hospotal Universitario "Dr. José E. González", Universidad Autónoma de Nuevo León, Monterrey, Nuevo León, México
}

\section{Resumen}

Antecedentes: Niveles alterados de cortisol se han asociado a un incremento en la mortalidad y disminución en la calidad de vida en pacientes con enfermedad renal crónica (ERC), sin embargo, la respuesta adrenal a la prueba de estimulación con adrenocorticotropina (ACTH) no ha sido evaluada en pacientes con ERC etapas 3a a 5 con y sin terapia de reemplazo renal (TRR). Objetivo: Evaluar la función adrenal de pacientes con ERC. Materiales y métodos: Adultos con ERC se sometieron a una prueba de estimulación con cosintropina a dosis baja (1 $\mu \mathrm{g}$ de ACTH sintética) y se midieron los niveles séricos de cortisol a los $0,+30$ y +60 minutos postestimulación. Resultados: 60 participantes con ERC en etapas 3, 4 y 5 (con y sin TRR) fueron incluidos. Ninguno de los pacientes presentó insuficiencia adrenal (IA). La correlación observada entre la concentración basal, a los 30 minutos y 1 hora de cortisol postestimulación y la tasa de filtrado glomerular (TFG) fue negativa y estadísticamente significativa ( $r:-0.39$ [ $p=0.002$ ], $r:-0.363$ [ $p=0.004], r:-0.4$ [p = 0.002], respectivamente). Conclusión: Desde etapas tempranas de la ERC los niveles de cortisol se incrementan a medida que la TFG disminuye. Concluimos que no es necesario un tamizaje sistemático para detectar IA en pacientes con ERC.

PALABRAS CLAVE: Cortisol. Insuficiencia adrenal. Enfermedad renal crónica.

\section{Adrenal functional reserve in the full spectrum of chronic kidney disease}

\section{Abstract}

Background: Altered cortisol levels have been associated with an increase in mortality and a decrease in health-related quality of life in patients with chronic kidney disease (CKD); however, adrenal response to adrenocorticotropic hormone (ACTH) stimulation test has not been evaluated in patients with stage 3a to 5 CKD with and without renal replacement therapy (RRT). Objective: To evaluate adrenal function in patients with CKD. Materials and methods: Adults with CKD underwent a low-dose cosyntropin stimulation test $(1 \mu \mathrm{g}$ synthetic $A C T H)$, with serum cortisol levels being measured at $0,+30$ and +60 minutes post-test. Results: Sixty participants with stage 3, 4 and 5 CKD (with and without RRT) were included. None of the patients had adrenal insufficiency (Al). The correlation observed between cortisol concentration at baseline and 30 minutes and 1 hour after stimulation and glomerular filtration rate (GFR) was negative and statistically significant ( $r:-0.39$ [p $=0.002], r:-0.363$ [ $p=0.004], r:-0.4$ [ $p=0.002]$, respectively). Conclusion: Since CKD early stages, cortisol levels increase as GFR decreases. Therefore, we conclude that systematic screening for $A l$ is not necessary in CKD patients.

KEY WORDS: Cortisol. Adrenal insufficiency. Chronic kidney disease.

Correspondencia:

*René Rodríguez-Gutiérrez

E-mail: rodriguezgutierrez.rene@mayo.edu
Gac Med Mex. 2021;157:519-524

Disponible en PubMed

www.gacetamedicademexico.com CC BY-NC-ND (http://creativecommons.org/licenses/by-nc-nd/4.0/). 


\section{Introducción}

La enfermedad renal crónica (ERC) es uno de los padecimientos crónicos más prevalentes y devastadores debido a la asociada disminución en la calidad de vida, morbilidad y mortalidad ${ }^{1,2}$. Se ha descrito que los pacientes con ERC presentan alteraciones a nivel del eje hipofisario-adrenal ${ }^{3-5}$ causando trastornos hormonales. De hecho, el desequilibrio en los niveles de aldosterona y cortisol se ha asociado a un incremento en la mortalidad y a una disminución de la calidad de vida relacionada con la salud en pacientes con ERC $^{5-7}$.

Los síntomas y las alteraciones metabólicas presentes tanto en la ERC como en la disfunción adrenal pueden sobreponerse. Además, en el contexto de ERC y otras comorbilidades la IA pudiera ser infradiagnosticada, incluso por médicos con experiencia.

Estudios previos han demostrado que los pacientes con ERC en etapa terminal presentan niveles de cortisol de normales a elevados posterior a una prueba de estimulación con cosintropina a dosis de entre 1 $\mu \mathrm{g}$ (dosis baja) a $250 \mu \mathrm{g}$ (dosis estándar). Sin embargo, dichos estudios se enfocaron en pacientes bajo terapia de reemplazo renal (TRR), incluyeron muestras pequeñas y las dosis empleadas de cosintropina variaban en cada estudio ${ }^{8-14}$. Adicionalmente, la reserva funcional adrenal no ha sido estudiada en pacientes con ERC etapas 3a a 5 con y sin TRR.

Por tal motivo, decidimos evaluar la función adrenal en pacientes del espectro completo de la ERC mediante una prueba de estimulación con cosintropina a dosis baja.

\section{Materiales y métodos}

\section{Participantes}

Se reclutaron de manera consecutiva adultos con diagnóstico previo de ERC etapas 3a a 5 según los criterios de la KDIGO (Kidney Disease: Improving Global Outcomes) $)^{15}$. De acuerdo con la tasa de filtrado glomerular (TFG) estimada mediante la ecuación CKD-EPI ${ }^{16}$ se dividió a los participantes en cuatro grupos: a) etapa 3, b) etapa 4, c) etapa 5 sin TRR, y d) etapa 5 con TRR.

Se excluyeron los pacientes con antecedente de enfermedad infecciosa aguda en los seis meses previos, enfermedad oncológica o tiroidea, así como pacientes con medicamentos que alteran el eje hipofisiario-adrenal. El protocolo de estudio fue aprobado por el Comité de Ética en Investigación del Hospital Dr. José E. González de la Universidad Autónoma de Nuevo León y se obtuvo el consentimiento informado de todos los participantes.

\section{Protocolo de estudio}

Tras realizar una historia clínica completa y confirmar un ayuno de al menos ocho horas, se administró un $1 \mu \mathrm{g}$ de adrenocorticotropina (ACTH) sintética (Cortrosyn ${ }^{\circledR}$, Amphastar Pharmaceuticals Inc., Rancho Cucamonga, CA, EE.UU.) en bolo y se obtuvieron muestras de sangre a los 0,30 y 60 minutos postestimulación para medir los niveles séricos de cortisol.

La insuficiencia adrenal (IA) se definió como un pico de cortisol menor a $496 \mathrm{nmol} / \mathrm{l}$ a los 30 o 60 minutos postestimulación.

\section{Mediciones}

La concentración de cortisol se midió mediante electro-quimioluminiscencia utilizando el analizador Cobas $^{\circledR}$ e-411 con el kit comercial de Roche (Roche, SA de CV). El coeficiente de variación intraprueba e interprueba fue del 1.23 y $1.57 \%$ respectivamente. Todas las muestras de sangre se centrifugaron y se almacenaron a una temperatura de $-20^{\circ} \mathrm{C}$ para posteriormente analizarlas simultáneamente.

\section{Análisis estadístico}

El tamaño de la muestra se calculó tomando en cuenta una diferencia de $8.6 \mu \mathrm{g} / \mathrm{dl}$ entre las medias del pico de cortisol de los pacientes con ERC bajo TRR y los pacientes con ERC sin TRR ${ }^{12}$. Para alcanzar un poder estadístico del $80 \%$ se estimó que se requerían al menos 12 pacientes de cada categoría de ERC. Se utilizaron medidas de tendencia central y dispersión para variables numéricas y frecuencias y porcentajes para las categóricas. Las comparaciones entre grupos de las variables numéricas paramétricas y no paramétricas se obtuvieron mediante las pruebas ANOVA y Kruskal-Wallis, respectivamente. Se condujeron análisis post hoc utilizando la corrección de Bonferroni. Para variables categóricas se utilizó la prueba de chi cuadrada de Pearson. El coeficiente de correlación de Pearson se utilizó para evaluar la correlación entre la TFG y la concentración sérica de cortisol. Un coeficiente de Pearson $>0.5$ se 
Tabla 1. Características basales de los pacientes en las diferentes etapas de la ERC*

\begin{tabular}{|c|c|c|c|c|c|}
\hline & Etapa 3 & Etapa 4 & Etapa $5 \sin$ TRR & Etapa 5 con TRR & valor $p$ \\
\hline Edad & $58(9.6)$ & $55.2(8.2)$ & $52.4(8.7)$ & $51.8(15)$ & 0.36 \\
\hline Sexo femenino & $9(60)$ & $5(33.3)$ & $6(40)$ & $7(46.7)$ & 0.5 \\
\hline Creatinina & $1.6(.42)$ & $3(.57)$ & $6.2(2.3)$ & $7.3(3.3)$ & $<0.001$ \\
\hline TFG & $39(7.8)$ & $21(4.2)$ & $9.8(3.9)$ & $8(3.3)$ & $<0.001$ \\
\hline Meses con ERC & $12(6-36)$ & $12(8-24)$ & $12(7-24)$ & $24(12-72)$ & 0.37 \\
\hline Peso & $75(15)$ & $79.4(21.5)$ & $76(15)$ & $71.2(21.6)$ & 0.6 \\
\hline IMC & $30(6.1)$ & $29.5(5.9)$ & $29.2(6.9)$ & $26.8(6.5)$ & 0.53 \\
\hline Presión arterial sistólica & $144(12.6)$ & $136(23)$ & $133.8(25.3)$ & $148.4(20.3)$ & 0.2 \\
\hline Presión arterial diastólica & $77.5(10.3)$ & $73.6(1)$ & $75.7(14.9)$ & $82.2(15.7)$ & 0.3 \\
\hline Hipertensión & $15(100)$ & $14(93.3)$ & $15(100)$ & $15(100)$ & 0.3 \\
\hline Diabetes mellitus & $11(73.3)$ & $11(73.3)$ & $13(86.7)$ & $14(93.3)$ & 0.3 \\
\hline Dislipidemia & $11(73.3)$ & $4(26.7)$ & $3(20)$ & $5(33.3)$ & 0.01 \\
\hline Infarto agudo de miocardio & $1(6.7)$ & $1(6.7)$ & $1(6.7)$ & $1(6.7)$ & 1 \\
\hline Evento cerebrovascular & $4(26.7)$ & $2(13.3)$ & $1(6.7)$ & 0 & 0.12 \\
\hline Insuficiencia cardiaca & $4(26.7)$ & $2(13.3)$ & $1(6.7)$ & $1(6.7)$ & 0.32 \\
\hline Antihipertensivos & $15(100)$ & $14(93.3)$ & $15(100)$ & $15(100)$ & 0.38 \\
\hline Agentes hipoglucemiantes & $3(20)$ & $1(6.7)$ & $1(6.7)$ & $1(6.7)$ & 0.52 \\
\hline Insulina & $8(53.3)$ & $9(60)$ & $10(66.7)$ & $8(53.3)$ & 0.86 \\
\hline Aspirina & $3(20)$ & $3(20)$ & $2(13.3)$ & $1(6.7)$ & 0.69 \\
\hline Estatinas & $12(80)$ & $10(66.7)$ & $8(53.3)$ & $2(13.3)$ & 0.002 \\
\hline Fibratos & $1(6.7)$ & $2(13.3)$ & 0 & $1(6.7)$ & 0.54 \\
\hline Diuréticos & $9(60)$ & $12(80)$ & $10(66.7)$ & $5(33.3)$ & 0.06 \\
\hline Hemoglobina & $11.6(1.5)$ & $11.1(1.7)$ & $9.8(1.3)$ & $9.8(2.53)$ & 0.014 \\
\hline Conteo de glóbulos blancos & $8.3(2.5)$ & $7.4(1.7)$ & $7.3(1.3)$ & $7.8(2.8)$ & 0.63 \\
\hline Neutrófilos & $5.1(2.5)$ & $4.3(1.2)$ & $4.7(1.1)$ & $5.5(2.3)$ & 0.36 \\
\hline Linfocitos & $2.2(.5)$ & $2.2(.8)$ & $1.6(.6)$ & $1.4(.6)$ & 0.001 \\
\hline Plaquetas & $240.80(64.79)$ & $235.86(57.68)$ & $218.53(56.72)$ & $194.6(99.7)$ & 0.29 \\
\hline Nitrógeno ureico en sangre & $25.20(14.71)$ & $40.13(15.09)$ & $64.16(22.66)$ & $38.51(16.69)$ & $<0.001$ \\
\hline Glucosa & 107 (93-138) & $116(90-131)$ & $111(101-127)$ & $136(107-159)$ & 0.25 \\
\hline Sodio & $138.4(3.1)$ & $137.3(4.4)$ & $137.3(4)$ & $135.3(5.5)$ & 0.27 \\
\hline Cloro & $104.6(4.9)$ & $106.6(3.6)$ & $106.2(5.9)$ & $97.6(5.7)$ & $<0.001$ \\
\hline Potasio & $4.5(.6)$ & $4.7(.7)$ & $4.8(.7)$ & $4(.6)$ & 0.007 \\
\hline Calcio & $8.7(.4)$ & $8.6(.7)$ & $8.2(.5)$ & $8.7(1.3)$ & 0.34 \\
\hline
\end{tabular}

Medidas expresadas como frecuencias (\%), media (desviación estándar) o mediana (rango intercuartil). tERC: enfermedad renal crónica; TRR: terapia de reemplazo renal; TFG: tasa de filtrado glomerular. 
Tabla 2. Valores de cortisol (nmol/l) antes y después de la administración de $1 \mu \mathrm{g}$ de Cortrosyn ${ }^{\circledR}$

\begin{tabular}{|l|c|c|c|c|c|}
\hline & Etapa 3 & Etapa 4 & Etapa 5 sin TRR & Etapa 5 con TRR & valor p \\
\hline Cortisol basal & $418.2(121.8)$ & $469(124)$ & $594.2(301.1)$ & $590.6(228)$ & 0.05 \\
\hline Cortisol a los 30 minutos & $825.6(165.3)$ & $828.4(98)$ & $957.1(305)$ & $971(201.8)$ & 0.09 \\
\hline Cortisol a 1 hora & $895.6(157.1)$ & $931.5(164.4)$ & $1083(342.6)$ & $1112.1(221.5)$ & 0.03 \\
\hline Pico & $915.3(164.8)$ & $943.2(146.7)$ & $1083(342.6)$ & $1112.1(221.5)$ & 0.05 \\
\hline Delta cortisol & $497(160.3)$ & $474.2(140.5)$ & $488.7(163.7)$ & $521.4(116.4)$ & 0.84 \\
\hline
\end{tabular}

*Valores expresados como media (desviación estándar).

TRR: terapia de reemplazo renal.

Tabla 3. Valores de cortisol (nmol/l) antes y después de administración de $1 \mu \mathrm{g}$ de Cortrosyn ${ }^{\circledast}$ en 3 grupos*

\begin{tabular}{|c|c|c|c|c|}
\hline & $\begin{array}{l}\text { Etapa } 3 \\
(n=15)\end{array}$ & $\begin{array}{l}\text { Etapa } 4 \\
(n=15)\end{array}$ & $\begin{array}{l}\text { Etapa } 5 \text { con y sin TRR } \\
\qquad(n=30)\end{array}$ & valor $p$ \\
\hline Cortisol basal & $418.2(121.8)$ & $469(124)$ & $592.4(262.4)$ & 0.021 \\
\hline Cortisol a los 30 minutos & $825.6(165.3)$ & $828.4(98)$ & $964.1(254.2)$ & 0.042 \\
\hline Cortisol a 1 hora & $895.6(157.1)$ & $931.5(164.4)$ & $1097.5(283.8)$ & 0.012 \\
\hline Pico & $915.3(164.8)$ & $943.2(146.7)$ & $1097.5(283.8)$ & 0.022 \\
\hline Delta cortisol & $497(160.3)$ & $474.2(140.5)$ & $505.1(140.6)$ & 0.79 \\
\hline
\end{tabular}

*Valores expresados como media (desviación estándar).

TRR: terapia de reemplazo renal.

estableció como evidencia de una correlación lineal de fuerza moderada. Un valor de $p<0.05$ se consideró estadísticamente significativo. El análisis se realizó utilizando el software IBM SPSS Statistics v25 (IBM 2019).

\section{Resultados}

Se incluyeron un total de 60 pacientes con ERC (15 en cada grupo). Las características basales se muestran en la tabla 1.

\section{Prevalencia de insuficiencia adrenal y comparación de cortisol sérico}

Ninguno de los pacientes incluidos mostró IA (Tabla 2). Sin embargo, se encontró una diferencia significativa en la concentración de cortisol plasmático a los 60 minutos postestimulación entre los cuatro grupos $(p=0.03)$. Se observó una tendencia de incremento en los niveles basales de cortisol a lo largo de las distintas categorías de ERC. Cuando se analizaron los pacientes en etapa 5 (con y sin TRR) como un mismo grupo, observamos una diferencia estadísticamente significativa en los picos de cortisol $(p=0.02)$, en la concentración basal $(p=0.02)$, a los $30(p=0.042)$ y 60 minutos $(p=0.012)$ entre los tres grupos (Tabla 3). La correlación observada entre la concentración basal de cortisol, a los 30 minutos y 1 hora de postestimulación y la TFG fue negativa y estadísticamente significativa $(r:-0.39[p=0.002], r:-0.363$ [p $=0.004], r:-0.4$ [p $=0.002]$, respectivamente).

La tabla 4 muestra los niveles de cortisol basales y postestimulación de acuerdo con los niveles séricos de nitrógeno ureico en la sangre.

\section{Discusión}

La literatura previa se había enfocado en la relación entre los niveles de cortisol y la TFG en vez de con la incidencia de IA en pacientes con ERC ${ }^{8-14}$. En nuestro estudio, ningún participante cumplió con los criterios de IA como respuesta a la prueba de estimulación con cosintropina a dosis baja $(1 \mu \mathrm{g})^{17}$. Sin embargo, encontramos diferencias significativas entre los grupos de ERC (etapa 3, 4 y 5 con y sin TRR), en los niveles de cortisol basal, a los 30 y a los 60 minutos postestimulación. 
Tabla 4. Valores de cortisol (nmol/l) antes y después de administración de $1 \mu \mathrm{g}$ de Cortrosyn ${ }^{\circledR}$ en grupos divididos de acuerdo con el nivel de nitrógeno ureico en sangre*

\begin{tabular}{|l|c|c|c|}
\hline & $<60$ & $>60$ & valor $p$ \\
\hline Cortisol basal & $479.8(167.4)$ & $688.3(321.7)$ & 0.01 \\
\hline Cortisol a los 30 minutos & $876.1(180.2)$ & $1022.4(299.4)$ & 0.84 \\
\hline Cortisol a 1 hora & $971.6(198.5)$ & $1156.7(371.4)$ & 0.04 \\
\hline Pico & $981.2(193.3)$ & $1156.7(371.4)$ & 0.033 \\
\hline Delta cortisol & $501.4(138)$ & $468.3(171.5)$ & 0.46 \\
\hline
\end{tabular}

*Valores expresados como media (desviación estándar).
ERC. Se observó una correlación negativa entre los niveles séricos de cortisol y la TFG, sin embargo, se requieren estudios adicionales para esclarecer la importancia clínica de esta asociación.

\section{Agradecimientos}

Los autores agradecen a Tomás Alvarado Peña, Patricio Guillermo García Espinoza, Rodolfo Alan Aguilar Torres y Edgar Botello Hernández por su valiosa participación en el estudio.

\section{Financiamiento}

La prevalencia de IA en pacientes bajo TRR que presentaban hipotensión fue del $26 \%$ en estudios en los que se midió el cortisol salival ${ }^{18-20}$. En nuestro estudio, la ausencia de IA podría ser explicada por la falta de pacientes con hipotensión y por la medición del cortisol sérico. Por tal motivo, sería valioso evaluar la concordancia entre los niveles séricos y salivales de cortisol en pacientes con ERC para validar el uso de este método como prueba de tamizaje para $I^{21}$.

Se espera que los niveles de cortisol aumenten en situaciones de estrés ${ }^{22}$, como lo es la ERC, lo cual podría explicarse por la disminución del aclaramiento renal y por la inactividad de la 11-beta-hidroxiesteroide-deshidrogenasa tipo $2^{23}$. Sin embargo, estudios en los que se incluyeron pacientes con ERC y controles sanos no encontraron diferencias estadísticamente significativas entre los niveles de cortisol salival ${ }^{9,14,18}$. Nuestros resultados demuestran que desde etapas tempranas de la ERC, la concentración de cortisol se incrementó a medida que la TFG iba disminuyendo, lo cual no había sido reportado previamente. Estos hallazgos sugieren un efecto protector contra IA en etapas terminales de $\mathrm{ERC}^{8-14}$.

Dentro de las fortalezas de nuestro estudio se encuentra la utilización de una muestra superior de pacientes pertenecientes al espectro completo de la ERC con pocas diferencias demográficas y bioquímicas entre los cuatro grupos, de las cuales ninguna ha sido relacionada previamente con una disfunción de la glándula adrenal ${ }^{24}$. Como limitación, la mayoría de nuestros pacientes tenían diabetes y eran relativamente jóvenes (50-55 años).

\section{Conclusiones}

Nuestros resultados sugieren que no es necesaria la evaluación de la función adrenal en pacientes con
Este trabajo fue financiado con recursos propios del Servicio de Endocrinología del Hospital Universitario Dr. José E. González de la Universidad Autónoma de Nuevo León.

\section{Conflicto de intereses}

Los autores declaran no tener ningún conflicto de intereses.

\section{Responsabilidades éticas}

Protección de personas y animales. Los autores declaran que los procedimientos seguidos se conformaron a las normas éticas del comité de experimentación humana responsable y de acuerdo con la Asociación Médica Mundial y la Declaración de Helsinki.

Confidencialidad de los datos. Los autores declaran que han seguido los protocolos de su centro de trabajo sobre la publicación de datos de pacientes.

Derecho a la privacidad y consentimiento informado. Los autores han obtenido el consentimiento informado de los pacientes y/o sujetos referidos en el artículo. Este documento obra en poder del autor de correspondencia.

\section{Bibliografía}

1. Hill NR, Fatoba ST, Oke JL, Hirst JA, Callaghan AO, Lasserson DS, et al. Global prevalence of chronic kidney disease - A systematic review and meta-analysis. PLoS One. 2016;11(7):1-18

2. GBD Chronic Kidney Disease Collaboration. Global, regional, and national burden of chronic kidney disease, 1990-2017: a systematic analysis for the Global Burden of Disease Study 2017. Lancet. 2020;395(10225):709-33.

3. Belarbia A, Nouira S, Sahtout W, Guedri Y, Achour A. Aldosterone renin ratio and chronic kidney disease. Saudi J Kidney Dis Transplant. 2015;26(5):931-40.

4. Asao T, Oki K, Yoneda M, Tanaka J, Kohno N. Hypothalamic-pituitary-adrenal axis activity is associated with the prevalence of chronic kidney disease in diabetic patients. Endocr J. 2016;63(2):119-26.

5. Meuwese CL, Carrero JJ. Chronic kidney disease and hypothalamic-pituitary axis dysfunction: The chicken or the egg? Arch Med Res. 2013;44(8):591-600 
6. Drechsler C, Ritz E, Tomaschitz A, Pilz S, Schönfeld S, Blouin K, et al. Aldosterone and cortisol affect the risk of sudden cardiac death in haemodialysis patients. Eur Heart J. 2013;34(8):578-85.

7. Ros S, Carrero JJ. Endocrine alterations and cardiovascular risk in CKD: Is there a link? Nefrologia. 2013:33(2):181-7.

8. Clodi M, Riedl M, Schmaldienst S, Vychytil A, Kotzmann H, Kaider A, et al. Adrenal function in patients with chronic renal failure. Am J Kidney Dis. 1998:32(1):52-5.

9. Grekas D, Tourkantonis A, Pharmakiotis A. Adrenal responsiveness during and after intermittent hemodialysis. Clin Exp Dial Apheresis. 1983;7(3):197-205.

10. McDonald WJ, Golper TA, Mass RD, Kendall JW, Porter GA, Girard DE et al. Adrenocorticotropin-cortisol axis abnormalities in hemodialysis patients. J Clin Endocrinol Metab. 1979;48(1):92-5.

11. Ramirez G. Evaluation of the hypothalamic hypophyseal adrenal axis in patients receiving long-term hemodialysis. Arch Intern Med. 1982 142(8):1448-52

12. Siamopoulos KC, Dardamanis M, Kyriaki D, Pappas M, Sferopoulos G, Alevisou V. Pituitary adrenal responsiveness to corticotropin-releasing hormone in chronic uremic patients. Perit Dial Int. 1990; 10(2):153-6.

13. Siamopoulos KC, Eleftheriades EG, Pappas M, Sferopoulos G, Tsolas O. Ovine corticotropin-releasing hormone stimulation test in patients with chronic renal failure: Pharmacokinetic properties, and plasma adrenocorticotropic hormone and serum cortisol responses. Horm Res Paediatr. 1988:30:17-21

14. Luger A, Lang I, Kovarik J, Stummvoll HK, Templ H. Abnormalities in the hypothalamic-pituitary-adrenocortical axis in patients with chronic renal failure. Am J Kidney Dis. 1987;9(1):51-4.
15. Kidney Disease: Improving Global Outcomes (KDIGO) CKD Work Group. KDIGO 2012 Clinical Practice Guideline for the Evaluation and Management of Chronic Kidney Disease. Kidney Int Suppl. 2013;3(1).

16. Levey AS, Stevens LA, Schmid CH, Zhang Y, Castro III AF, Feldman HI, et al. A new equation to estimate glomerular filtration rate disclosure of conflicts of interest: We have received confirmation from Drs. Ann Intern Med. 2009;150(9):604-12.

17. Bornstein SR, Allolio B, Arlt W, Barthel A, Don-Wauchope A, Hammer GD, et al. Diagnosis and treatment of primary adrenal insufficiency: An endocrine society clinical practice guideline. J Clin Endocrinol Metab. 2016;101(2):364-89.

18. Arregger AL, Cardoso EML, Zucchini A, Aguirre EC, Elbert A, Contreras $L N$. Adrenocortical function in hypotensive patients with end stage renal disease. Steroids. 2014;84:57-63.

19. Arregger AL, Cardoso EML, Tumilasci O, Contreras LN. Diagnostic value of salivary cortisol in end stage renal disease. Steroids. 2008.73(1):77-82.

20. Harvey CJ, Gower PE, Hawkins PN, Pepys MB, Phillips ME. Occult adrenal insufficiency secondary to amyloidosis in the context of chronic renal failure. Nephrol Dial Transplant. 1995;10(7):1237-9.

21. Bastin P, Maiter D, Gruson D. Salivary cortisol testing: Preanalytic and analytic aspects. Ann Biol Clin (Paris). 2018;76(4):393-405.

22. Elbuken G, Karaca Z, Tanriverdi F, Unluhizarci K, Kelestimur F. Assessment of the hypothalamic-pituitary-adrenal axis in critical illness. Expert Rev Endocrinol Metab. 2011;6(1):35-48.

23. N'Gankam V, Uehlinger D, Dick B, Frey BM, Frey FJ. Increased cortisol metabolites and reduced activity of $11 \beta$-hydroxysteroid dehydrogenase in patients on hemodialysis. Kidney Int. 2002:61(5):1859-66.

24. Mazziotti G, Formenti AM, Frara S, Roca E, Mortini P, Berruti A, et al. Management of endocrine disease risk of overtreatment in patients with adrenal insufficiency: Current and emerging aspects. Eur J Endocrinol. 2017;177(5):R231-48. 